\title{
Internações por condições sensíveis à atenção primária à saúde, 2008-2015: uma análise do impacto da expansão da ESF na cidade do Rio de Janeiro
}

\author{
Admissions for conditions sensitive to primary health care, \\ 2008-2015: an analysis of the impact of the expansion of the Family \\ Health Strategy in the city of Rio de Janeiro
}

\author{
Laís Pimenta Ribeiro dos Santos ${ }^{1}$, Ana Luisa Barros de Castro², \\ Viviane Gomes Parreira Dutra'1, Raphael Mendonça Guimarães ${ }^{3}$
}

\begin{abstract}
Resumo
Introdução: O município do Rio de Janeiro apresentou uma importante expansão da cobertura da Atenção Primária em Saúde nos moldes da Estratégia de Saúde da Família a partir de 2009, e é importante a avaliação sobre o impacto desta agenda. As internações por Condições Sensíveis à atenção primária são indicadores hospitalares utilizados como forma de avaliação da efetividade da APS. Objetivo: Avaliar a repercussão da expansão da Estratégia Saúde da Família (ESF) na internação por condições sensíveis à atenção primária (ICSAP) no período de 2008 a 2015. Método: Foram analisados indicadores de cobertura pela ESF e de ICSAP entre 2008 e 2015 no município do Rio de Janeiro. Obteve-se a correlação entre os indicadores por meio do coeficiente de Spearmann e calculou-se a razão de taxas, tendo como parâmetro o ano imediatamente anterior à expansão da cobertura no município (2008). Resultados: Identificou-se tendência ao aumento da cobertura da ESF e à redução dos indicadores de ICSAP, bem como correlação inversa entre a cobertura pela ESF e a proporção de ICSAP $(r=-0,888, p=0,020)$, e entre a cobertura pela ESF e a taxa de ICSAP $(r=-0,753, p=0,031)$. Observou-se associação significativa para as razões de taxa dos indicadores de cobertura a partir de 2011 e de taxas de internação a partir de 2013. Conclusão: A expansão da APS na cidade do Rio de Janeiro impactou positivamente nas internações por condições sensíveis à APS. É necessário discutir a padronização de serviços para as unidades de atenção primária e o estímulo à qualificação dos profissionais na área.
\end{abstract}

Palavras-chave: atenção primária à saúde; séries temporais; hospitalização; indicadores básicos de saúde; sistemas de saúde.

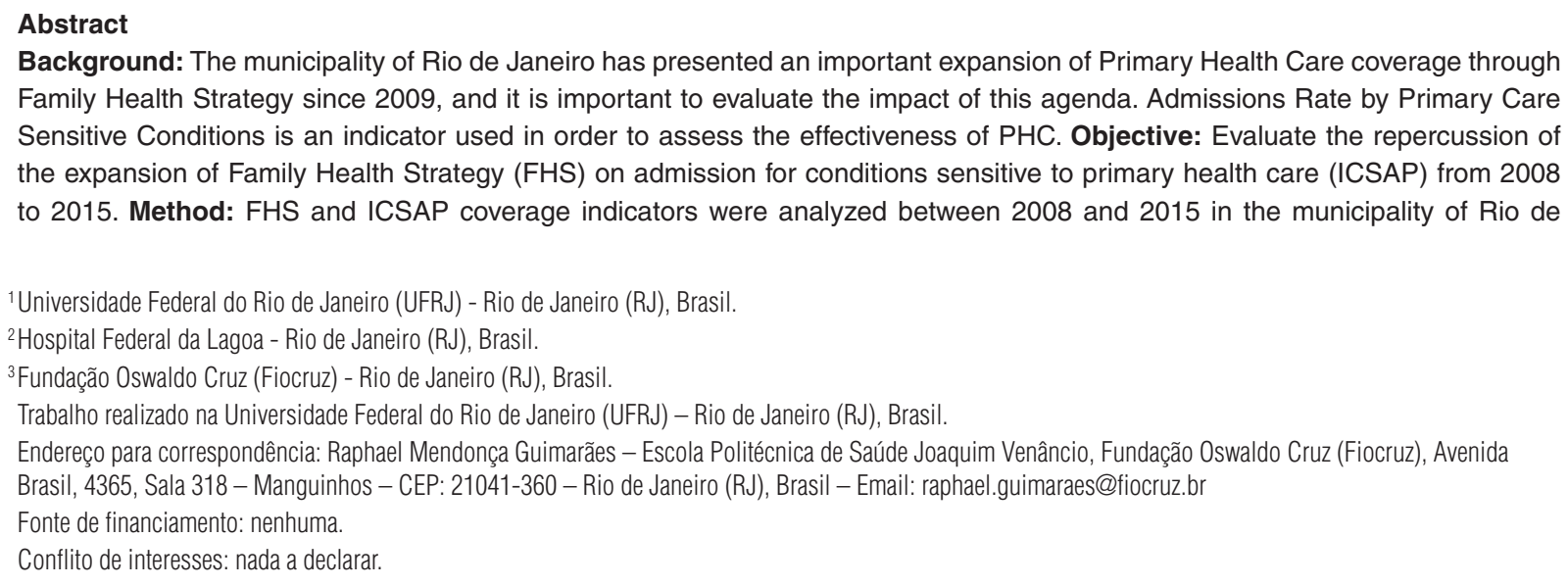

Background: The municipality of Rio de Janeiro has presented an important expansion of Primary Health Care coverage through Family Health Strategy since 2009, and it is important to evaluate the impact of this agenda. Admissions Rate by Primary Care Sensitive Conditions is an indicator used in order to assess the effectiveness of PHC. Objective: Evaluate the repercussion of the expansion of Family Health Strategy (FHS) on admission for conditions sensitive to primary health care (ICSAP) from 2008 to 2015. Method: FHS and ICSAP coverage indicators were analyzed between 2008 and 2015 in the municipality of Rio de 
Janeiro. Correlation between the indicators was obtained using Spearman's coefficient and the rate ratio was calculated using as a parameter the year immediately preceding the expansion of coverage in the municipality (2008). Results: Results identified a trend towards increased FHS coverage and reduced ICSAP indicators, inverse correlation between FHS coverage and the proportion of hospitalizations due to ICSAP $(r=-0.888, p=0.020)$, and between FHS coverage and ICSAP rate $(r=-0.753, p=0.031)$. Significant correlation was observed between the rate ratios of coverage indicators as of 2011 and the hospitalization rates as of 2013. Conclusion: Expansion of PHC in the city of Rio de Janeiro had a positive impact on hospitalizations because of conditions that were sensitive to PHC. It is necessary to discuss the standardization of services for PHC units and the encouragement of the qualification of professionals in the area.

Keywords: primary health care; time series; hospitalization; basic health indicators; health systems.

\section{INTRODUÇÃO}

A Atenção Primária à Saúde (APS) é um modelo de atenção à saúde apresentado pela Organização Mundial de Saúde como estratégia para ampliar o acesso e a qualidade da assistência às populações, visando à melhoria dos indicadores populacionais de saúde, especialmente à redução da morbimortalidade, e a um consumo mais racional da tecnologia biomédica, conferindo maior eficiência ao gasto no setor ${ }^{1,2}$.

Após a criação do Sistema Único de Saúde (SUS), foram iniciados diversos movimentos em busca da organização da assistência à saúde, de forma a proporcionar um cuidado mais efetivo para a população. Nesse contexto, em 2006, a Política Nacional de Atenção Básica forjou o termo Estratégia Saúde da Família (ESF) e definiu seu papel de orientador da APS praticada no país, configurando-se a principal porta de entrada e da Rede de Atenção à Saúde (RAS) $)^{3,4}$.

As condições sensíveis à atenção primária (CSAP) são um grupo de doenças para o qual a efetiva atuação da APS diminui o risco de internação $0^{5}$. As primeiras análises sobre essas condições foram realizadas por Billings e Teicholz ${ }^{6}$, 1990, em Columbia, nos Estados Unidos, que buscavam compreender o impacto do serviço ambulatorial nas internações hospitalares, sendo denominadas ambulatory care sensitive conditions. Posteriormente, esse indicador começou a ser utilizado no Canadá e em outros países com acesso universal para comparar a efetividade de modelos de $\mathrm{APS}^{2}$. No Brasil, a lista foi construída com base no perfil da população e contém 19 grupos de condições 7 .

A cidade do Rio de Janeiro experimentou um aumento vertiginoso da cobertura da ESF a partir da mudança de gestão, apresentando uma cobertura de 6,94\% em janeiro de 2009 para $46,16 \%$ em dezembro de $2015^{8}$. Esse aumento foi acompanhado de mudanças na organização dos serviços e no estímulo à qualificação dos serviços e da formação de profissionais para atuação nesse nível de atenção à saúde ${ }^{9}$. Em 2014, houve avaliação qualitativa dessa expansão com o instrumento PCATool, validado no Brasil, que identificou resultados melhores em locais do Rio de Janeiro, onde a proporção de turmas de Residência em Medicina de Família e Comunidade eram maiores ${ }^{10,11}$.

Este estudo tem como objetivo avaliar a repercussão da expansão da ESF na internação por condições sensíveis à atenção primária (ICSAP) no período de 2008 a 2015, com o intuito de discutir o impacto da expansão da APS nesse indicador hospitalar e utilizá-lo como um método de avaliação desse nível de atenção na cidade estudada. Além disso, esta pesquisa pode contribuir para a informação em saúde sobre a APS, de forma a subsidiar tomadas de decisão de gestores e também qualificar a discussão nas instituições de ensino em saúde para a formação de profissionais que atendam às necessidades desse modelo de atenção à saúde.

\section{MÉTODO}

Trata-se de estudo ecológico que analisou a internação por condições sensíveis à atenção primária (ICSAP) entre $2008 \mathrm{e}$ 2015 no município do Rio de Janeiro. Na análise, foram avaliados os conjuntos de doenças, classificadas de acordo com a Lista de Condições Sensíveis à Atenção Primária ${ }^{7}$ Os dados referentes à internação foram obtidos a partir do Sistema de Informações Hospitalares (SIH/SUS).

Inicialmente, foram calculados o número médio de internações e a taxa anual, segundo a Equação 1 a seguir:

$T x_{I C S A P}=\frac{\text { número de ICSAP }}{\text { habitantes }} \times 10.000$

Primeiramente, foi elaborada a série histórica do período por meio de gráficos de linha. Em seguida, calculou-se a tendência para internação pelos grupos de causa na população carioca. As tendências foram analisadas pelo modelo polinomial, cuja variável dependente $(\mathrm{Y})$ é representada pelas taxas de internação, e a variável independente $(\mathrm{X})$, pelos anos-calendário. Os seguintes modelos de regressão foram testados: linear $\left(y=\beta_{0}+\beta_{I} X\right)$ quadrático $\left(Y=\beta_{0}+\beta_{I} X+\beta_{2} X^{2}\right)$; e cúbico $\left(y=\beta_{0}+\beta_{I} X+\beta_{2} X^{2}+\beta_{3} X^{3}\right)$.

A fim de corrigir a potencial multicolinearidade entre os termos dos modelos, optou-se por trabalhar com a variável independente (o ano centralizado), ou seja, o ponto médio da série histórica. Dessa forma, para os anos entre 2008 e 2015, o termo $(\mathrm{x}$ - 2011) representou a variável ano centralizado, de acordo com a seguinte formalização do modelo (a exemplo do modelo linear testado, Equação 2):

$y_{T}=\beta_{0}+\beta_{1}(x-2011)$

em que: $\mathrm{y}_{\mathrm{T}}$ é a taxa de internação; $\mathrm{X}$, o ano; $\beta_{0}$, o coeficiente médio do período; e $\beta_{1}$, o incremento médio. 
Foi realizada a análise de resíduos, assim como foram avaliadas, para todos os modelos, a suposição de homocedasticidade e a aderência à distribuição normal. O critério de escolha do melhor modelo foi o valor do coeficiente de determinação $\left(\mathrm{R}^{2}\right)$ e da análise dos resíduos (especialmente para ratificação da suposição de homocedasticidade). Obedeceu-se ao critério de parcimônia para a escolha do modelo, ou seja, sempre pelo modelo mais simples com melhor poder explicativo. Considerou-se o modelo significativo sempre que o valor de $\mathrm{p}$ fosse inferior a 0,05 .

A fim de avaliar a correlação entre os indicadores de internação e os de cobertura pela ESF nos anos de estudo, foram calculados os coeficientes de correlação de Spearmann, uma vez que não se pode supor a relação de linearidade entre as variáveis nem a suposição de normalidade das variáveis.

O coeficiente de correlação de Spearmann $(\rho)$ mede o grau da correlação entre duas variáveis quantitativas, sem que haja a suposição de distribuição normal de nenhuma das duas variáveis. Esse coeficiente $(\rho)$ assume apenas valores entre -1 (relação perfeita e inversamente proporcional) $e+1$ (relação perfeita e diretamente proporcional). Quando $\rho=0$, diz-se que as variáveis são independentes. O coeficiente é dado por (Equação 3):

$\rho=\frac{6 \sum_{l} d_{i}^{2}}{\left(n^{3}-n\right)}$

em que: $\mathrm{n}$ é o número de pares $\left(\mathrm{x}_{\mathrm{i}}, \mathrm{y}_{\mathrm{i}}\right)$; e $\mathrm{d}_{\mathrm{i}}$, o (posto de $\mathrm{x}_{\mathrm{i}}$ dentro dos valores de $\mathrm{x}$ ) - (posto de $\mathrm{y}_{\mathrm{i}}$ dentro dos valores de $\mathrm{y}$ ).

Para se avaliar um possível impacto da expansão da cobertura da ESF, iniciada em 2009, na ocorrência das ICSAP, foram estimadas as taxas anuais de internação entre 2008 e 2015 para a totalidade de causas. Adotando-se o ano de 2008 como referência, por se tratar do ano imediatamente anterior à expansão, foram calculados as razões de taxa para cada ano e os seus respectivos intervalos de confiança, conforme a Equação 4 a seguir:

$$
\text { Razão de Taxas }(R T)=\frac{T I_{i}}{T I_{2008}}
$$

em que: $T I_{2008}$ é a taxa de internação pelo grupo de causa A em 2008; e $T I_{i}$, a taxa de internação pelo grupo de causa A no ano $i$.

Os dados foram analisados utilizando-se o programa estatístico $\mathrm{R}$, versão 3.1.2. Este estudo não foi submetido ao Comitê de Ética em Pesquisa por utilizar dados secundários de domínio público. Entretanto, foram respeitadas as normas vigentes no
Brasil, relacionadas à ética na pesquisa com seres humanos, de acordo com a Resolução nº 466/2012.

\section{RESULTADOS}

A série histórica de cobertura pela ESF e os indicadores de condições sensíveis à atenção primária (proporção de internações e taxa de internação) indicam que há um aumento na cobertura no período analisado e, concomitantemente, uma redução nas ICSAP, ambos com tendência significativa, conforme se observa na Tabela 1.

Ao tomar o ano de 2008 como referência (devido ao fato de ter sido o ano anterior à expansão da cobertura), observa-se que a expansão obteve um aumento estatisticamente significativo após 2011, mantendo-se crescente até 2015. Já a proporção de ICSAP decresceu de forma significativa a partir de 2013, enquanto a taxa de internações mostrou-se reduzida a partir de 2014. É importante notar que há uma correlação inversa entre a cobertura pela ESF e a proporção de $\operatorname{ICSAP}(\mathrm{r}=-0,888, \mathrm{p}=0,020)$ e entre a cobertura pela $\operatorname{ESF}$ e a taxa de $\operatorname{ICSAP}(r=-0,753, p=0,031)$ (Figura 1$)$. Isso mostra que, apesar de a redução estatisticamente significativa dos indicadores de internação não ocorrer paralelamente ao aumento estatisticamente significativo da cobertura (conforme observado na Tabela 2), há uma correlação entre esses indicadores, sugerindo haver um atraso entre a expansão da cobertura e o impacto real nos indicadores de saúde locais, demonstrando que o impacto da expansão da cobertura não é imediato.

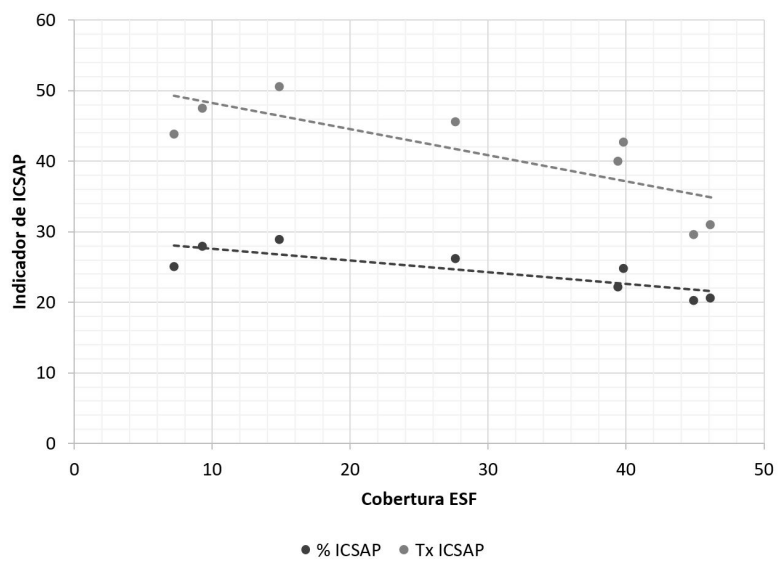

Figura 1. Correlação entre a cobertura de ESF e os indicadores de ICSAP no município do Rio de Janeiro, 2008-2015. Legenda: ESF = Estratégia Saúde da Família; \% ICSAP = Proporção de Internações por Condições Sensíveis à Atenção Primária; Tx ICSAP = Taxa de Internações por Condições Sensíveis à Atenção Primária

Tabela 1. Tendência para indicadores de cobertura e internações por condições sensíveis à atenção primária no município do Rio de Janeiro, 2008-2015

\begin{tabular}{|c|c|c|c|c|}
\hline Indicador & Equação & $\mathbf{R}^{2}$ & p valor & Tendência \\
\hline Cobertura ESF & $y=6,4 x+8,64$ & 0,94 & $<0,001$ & Crescente \\
\hline \% ICSAP & $y=-0,9 x+24,48$ & 0,72 & 0,008 & Decrescente \\
\hline Tx. ICSAP & $y=-2,1 x+41,35$ & 0,70 & 0,009 & Decrescente \\
\hline
\end{tabular}

Legenda: ESF = Estratégia Saúde da Família; \% ICSAP = Proporção de Internações por Condições Sensíveis à Atenção Primária; Tx ICSAP = Taxa de Internações por Condições Sensíveis à Atenção Primária; $\mathrm{R}^{2}=$ Coeficiente de determinação 
Tabela 2. Razão de taxas para associação temporal de indicadores de cobertura e internações por condições sensíveis à atenção primária no município do Rio de Janeiro, 2008-2015

\begin{tabular}{|c|c|c|c|c|c|c|c|c|c|}
\hline \multirow{2}{*}{ Ano } & \multicolumn{3}{|c|}{ Cobertura ESF } & \multicolumn{3}{|c|}{$\%$ ICSAP } & \multicolumn{3}{|c|}{ Tx. ICSAP } \\
\hline & Indicador & RT & IC $95 \%$ & Indicador & RT & IC 95\% & Indicador & RT & IC 95\% \\
\hline 2008 & 7,20 & 1 & - & 25,12 & 1 & - & 43,80 & 1 & - \\
\hline 2009 & 9,24 & 1,07 & $0,61-1,53$ & 27,99 & 1,11 & $1,01-1,22$ & 47,59 & 1,09 & $0,96-1,22$ \\
\hline 2010 & 14,83 & 1,35 & $0,77-1,94$ & 28,96 & 1,15 & $1,05-1,26$ & 50,58 & 1,15 & $1,02-1,29$ \\
\hline 2011 & 27,62 & 1,87 & $1,07-2,67$ & 26,14 & 1,04 & $0,95-1,13$ & 45,59 & 1,04 & $0,92-1,17$ \\
\hline 2012 & 39,84 & 2,33 & $1,33-3,33$ & 24,70 & 0,98 & $0,89-1,07$ & 42,66 & 0,97 & $0,86-1,09$ \\
\hline 2013 & 39,41 & 2,22 & $1,27-3,18$ & 22,21 & 0,88 & $0,80-0,96$ & 39,98 & 0,91 & $0,80-1,02$ \\
\hline 2014 & 44,86 & 2,43 & $1,38-3,47$ & 20,26 & 0,81 & $0,73-0,88$ & 29,64 & 0,68 & $0,60-0,76$ \\
\hline 2015 & 46,16 & 2,45 & $1,40-3,51$ & 20,53 & 0,82 & $0,74-0,89$ & 30,95 & 0,71 & $0,62-0,79$ \\
\hline
\end{tabular}

Legenda: ESF = Estratégia Saúde da Família; \% ICSAP = Proporção de Internações por Condições Sensíveis à Atenção Primária; Tx ICSAP = Taxa de Internações por Condições Sensíveis à Atenção Primária; RT = Razão de Taxas; IC 95\% = Intervalo de confiança (95\%)

\section{DISCUSSÃO}

Os resultados obtidos no presente estudo demonstram uma tendência significativa para redução das ICSAP com o aumento da cobertura da APS nos moldes de ESF na cidade do Rio de Janeiro a partir do período analisado. É importante destacar que, no período de 2008 a 2015, a taxa de ICSAP reduziu de 43,80 para 30,95 por 10 mil habitantes, com queda mais acentuada a partir do ano de 2013, quando a cobertura da ESF já alcançava $39,5 \%{ }^{8,9}$. Ainda, o decréscimo da proporção de ICSAP a partir de 2013 e a redução na taxa de internações a partir de 2014, de forma significativa, correspondem ao período subsequente ao de consolidação da ESF na cidade do Rio de Janeiro ${ }^{10,11}$.

O declínio dos valores desse indicador de qualidade de assistência à saúde com foco na atenção primária acompanha os estudos realizados no Brasil e no mundo, além de sugerir que a lista brasileira contém condições que de fato podem ser tratadas nesse nível de atenção, prevenindo agravos que geram internações ${ }^{12-16}$. Destaca-se, nessa direção, a redução de $38,1 \%$ na cidade de Florianópolis em um período um pouco menor, de 2005 a 2011, no qual ocorreu o mesmo movimento de expansão da APS, indo de 61,7 a 89,3\% de cobertura de ESF no período analisado e chegando a $100 \%$ de cobertura no ano de $2015^{16}$. Portanto, observa-se que, onde a expansão da APS foi adotada como estratégia para reorganizar e ampliar o acesso à saúde pública, houve impactos positivos significativos nos indicadores de avaliação sobre as ICSAP ${ }^{16,17}$.

É preciso, contudo, ressaltar que, no ano de 2013, o total de ICSAP no Estado do Rio de Janeiro representava 19,68\% do total de internações hospitalares ${ }^{18}$, enquanto o resultado encontrado em países mais desenvolvidos e que também possuem acesso universal e sistema de saúde ordenado pela APS, como Espanha e Austrália, é de 7 a 13\% do total de internações para esse mesmo indicador ${ }^{4,19}$. Conforme descrito, há efeito de tempo a ser considerado, que descreve um atraso entre a expansão da cobertura e a redução nas ICSAP. Isso significa que o efeito, sobremaneira, ocorre em médio e longo prazo. Estudos no Sul do Brasil destacam igualmente essa relação, argumentando que, provavelmente, a cobertura de ESF não foi suficiente para impactar as ICSAP ${ }^{20}$. Atentam, ainda, para o fato de que, apesar dos incrementos nos investimentos financeiros e na cobertura populacional pela ESF, ambos ainda não alcançaram níveis para garantir cuidados de saúde adequados à população ${ }^{21}$.

Outro aspecto que merece avaliação é o investimento da gestão na qualificação dos profissionais e no estabelecimento de padrões e de rotinas nos atendimentos, com a criação, por exemplo, da Carteira de Serviços em 2011, com a relação de serviços prestados na APS, com o Protocolo de Enfermagem em Atenção Primária à Saúde de 2012, com os Guias Rápidos da Subsecretaria de Atenção Primária, Vigilância e Promoção da Saúde (SUBPAV) de 2012 e com a implantação da Residência em Medicina de Família e Comunidade (MFC) na cidade do Rio de Janeiro também no ano de $2012^{9}$.

Ainda, entre outras ações no período, destacam-se: a) o pagamento de remuneração variável por performance, que é uma modalidade em que as equipes recebem esse benefício de acordo com a qualidade da informação dos serviços realizados para as linhas de cuidado, além de ocorrer a implantação de prontuários eletrônicos em todas as unidades tipo $\mathrm{A}$, agilizando atendimentos e melhorando a qualidade e o padrão de registro dos profissionais de nível superior no modelo de Registro Orientado por Problemas (ROP), organizado em Subjetivo, Objetivo, Avaliação e Plano (SOAP) ${ }^{22}$; b) adesão ao Programa Nacional de Melhoria do Acesso e da Qualidade da Atenção Básica (PMAQ), cuja adesão é voluntária; e c) estímulo ao Certificado do Reconhecimento do Cuidado com Qualidade (CRCQ), havendo certificação de unidades em diversas áreas, o que pode aumentar o interesse dos profissionais atuantes em se qualificar e buscar melhorias nos processos de trabalho ${ }^{23,24}$.

Finalmente, vale mencionar que somente a expansão desse nível de atenção possivelmente não reduzirá esse indicador, sem que haja mudança em outros aspectos. Sobretudo, é importante que a APS possua os recursos para que assegurem 
seu funcionamento em um ambiente compatível com o alcance dos objetivos e metas estabelecidos ${ }^{18}$. Nesse sentido, cabe discutir a efetividade e a qualidade da APS, tendo como norte a formação e a qualificação dos profissionais para atuação nesse nível de atenção, os processos ainda individualizados e fragmentados, as fragilidades no sistema de referência e de contrarreferência, a elevada rotatividade dos profissionais, em especial do médico, e o distanciamento entre a atenção especializada e hospitalar e a realidade da APS na rede que compõe ${ }^{25}$. A esse respeito, Rehem ${ }^{26}$ cita que o conhecimento e a percepção dos profissionais atuantes na APS sobre as ICSAP sugerem limitação para sua utilização na avaliação desse nível de atenção. Em outras palavras, por ser um indicador hospitalar, com sua lista brasileira construída pouco tempo após a Política Nacional de Atenção Básica (PNAB) de 2006, existem limites para sua utilização como parâmetro único de avaliação da APS, em que os atributos essenciais e derivados devem se desdobrar em diversas atuações da equipe multidisciplinar, a qual necessita convergir saberes para o cuidado integral dos usuários. Nesse sentido, é importante adotar estratégias que garantam a boa qualidade da informação, com a criação de rotinas e de programações estatísticas que sejam funcionais e que garantam agilidade, precisão e validade dos dados ${ }^{27}$.

A partir do estudo realizado, foi possível identificar que a expansão da APS na cidade do Rio de Janeiro, assim como esperado a partir dos estudos realizados em outros locais, impactou positivamente nas internações por condições sensíveis à APS, demonstrando uma tendência para redução tanto na taxa quanto na proporção desse indicador. Dessa forma, identificou-se que é possível utilizar esse indicador como um dos parâmetros de avaliação de impacto em um sistema organizado a partir da APS no Brasil, o que vai ao encontro de propostas de organização de sistemas de saúde que estão sendo implantadas em diversos países, permitindo, a partir de análise de dados brutos, a legitimação da lista brasileira.

Sobretudo para a avaliação da qualidade da assistência da APS, seriam necessários outros modelos de avaliação, considerando que o impacto não é diretamente proporcional e que existem questões sociais, econômicas e de processo de trabalho que influenciam o indicador estudado, ou seja, os atributos e a efetividade da APS não podem ser analisados e discutidos partindo unicamente dessas internações.

Ademais, foi possível identificar que a expansão da APS necessita de outras tomadas de decisão para aumentar a sua efetividade e o impacto no indicador estudado, como a padronização de serviços para as unidades de atenção primária e o estímulo à qualificação dos profissionais que atuam na área.

Conclui-se que o estudo colabora, de forma significativa, para a tomada de decisão de gestores, para o olhar dos profissionais de saúde dos diferentes níveis de atenção, educadores e estudantes da área da saúde, pois norteia para os impactos esperados e reforça a importância da discussão dos modelos de atenção e da formação de profissionais para a atuação em saúde pública, justificados pelo real impacto da APS em um sistema de saúde.

\section{REFERÊNCIAS}

1. World Health Organization. Declaration of Alma-Ata International Conference on Primary Health Care, Alma-Ata, USSR [Internet]. Geneva: WHO; 1978 [citado em 2016 maio 20]. Disponível em: http://www.who. int/publications/almaata_declaration_en.pdf

2. Nedel FB. Internações hospitalares evitáveis pela atenção primária: estudo do impacto do Programa de Saúde da Família sobre as internações por Condições Sensíveis à Atenção Primária no Rio Grande do Sul, Brasil [tese]. Rio Grande do Sul: Programa de Pós-graduação em Epidemiologia, Universidade Federal de Pelotas; 2009 [citado em 2016 maio 20]. Disponível em: http://www.epidemio-ufpel.org.br/uploads/teses/tese\%20nedel.pdf

3. Mendes EV. As redes de atenção à saúde [Internet]. Brasília: Organização Pan-Americana da Saúde; 2011 [citado 2016 junho 10]. Disponível em: http://www.conass.org.br/biblioteca/pdf/A-Atencao-Primaria-e-as-Redesde-Atencao-a-Saude.pdf

4. Castro ALB, Andrade CLT, Machado CV, Lima LD. Condições socioeconômicas, oferta de médicos e internações por condições sensíveis à atenção primária em grandes municípios do Brasil. Cad Saude Publica. 2015;31(11):2353-66. http://dx.doi.org/10.1590/0102-311X00126114. PMid:26840815.

5. Alfradique ME, Bonolo PF, Dourado I, Lima-Costa MF, Macinko J, Mendonça CS, et al. Internações por condições sensíveis à atenção primária: a construção da lista brasileira como ferramenta para medir o desempenho do sistema de saúde (Projeto ICSAP - Brasil). Cad Saude Publica. 2009;25(6):1337-49. http://dx.doi.org/10.1590/S0102-311X2009000600016. PMid:19503964.

6. Billings J, Teicholz N. Uninsured patients in District of Columbia Hospitals. Health Aff (Millwood). 1990;9(4):158-65. http://dx.doi.org/10.1377/ hlthaff.9.4.158. PMid:2289752.

7. Brasil. Ministério da Saúde. Secretaria de Atenção à Saúde. Portaria nº 221, de 17 de abril de 2008. Diário Oficial da União [Internet], Brasília, 18 de abril de 2008 [citado 2016 junho 10]. Disponível em: http://bvsms.saude. gov.br/bvs/saudelegis/sas/2008/prt0221_17_04_2008.html

8. Brasil. Ministério da Saúde. Departamento de Atenção Básica. Histórico de cobertura da saúde da família [Internet]. Brasília; 2016 [citado 2016 junho 10]. Disponível em: http://dab.saude.gov.br/portaldab/historico_ cobertura_sf.php

9. Soranz D, Pinto LF, Penna GO. Eixos e a Reforma dos Cuidados em Atenção Primária em Saúde (RCAPS) na cidade do Rio de Janeiro, Brasil. Cien Saude Colet. 2016;21(5):1327-38. http://dx.doi.org/10.1590/141381232015215.01022016. PMid:27166884.

10. Brasil. Portaria $\mathrm{n}^{\circ} 4.279$, de 30 de dezembro de 2010. Diretrizes para a organização da Rede de Atenção à Saúde no âmbito do Sistema Único de Saúde. Diário Oficial da União [Internet], Brasília, 31 de dezembro de 2010 [citado 2016 junho 10]. Disponível em: http://conselho.saude.gov. br/ultimas_noticias/2011/img/07_jan_portaria4279_301210.pdf

11. Harzheim E, Hauser L, Pinto LF. Avaliação do grau de orientação para Atenção Primária em Saúde: a experiência dos usuários das Clínicas da 
Família e Centros Municipais de Saúde na cidade do Rio de Janeiro. Rio Grande do Sul: Faculdade de Medicina, Universidade Federal do Rio Grande do Sul; 2015. Relatório Final de Pesquisa PCATool Rio2014.

12. Dias-da-Costa JS, Büttenbender DC, Hoefel AL, Souza LL. Hospitalizações por condições sensíveis à atenção primária nos municípios em gestão plena do sistema no Estado do Rio Grande do Sul, Brasil. Cad Saude Publica. 2010;26(2):358-64. http://dx.doi.org/10.1590/S0102-311X2010000200014. PMid:20396850.

13. Pazó RG, Frauches DO, Galvêas DP, Stefenoni AV, Cavalcante ELB, Pereira-Silva FH. Internações por condições sensíveis à atenção primária no Espírito Santo: estudo ecológico descritivo no período 2005-2009. Revista Epidemiologia e Serviços de Saúde. 2012;21(2):275-82. http:// dx.doi.org/10.5123/S1679-49742012000200010.

14. Mendonça SS, Albuquerque EC. Perfil das internações por condições sensíveis à atenção primária em Pernambuco, 2008 a 2012. Revista Epidemiologia e Serviços de Saúde. 2014;23(3):463-74. http://dx.doi.org/10.5123/S167949742014000300009 .

15. Pereira FJR, Silva CC, Lima EA No. Condições Sensíveis à Atenção Primária: uma revisão descritiva dos resultados da produção acadêmica brasileira. Saúde em Debate. 2014;38:331-42.

16. Brasil VP, Costa JSD. Hospitalizações por condições sensíveis à atenção primária em Florianópolis, Santa Catarina: estudo ecológico de 2001 a 2011. Epidemiol Serv Saude. 2016;25(1):75-84. PMid:27861680.

17. Instituto Brasileiro de Geografia e Estatística. Censo demográfico: características gerais da população, religião e pessoas com deficiência [Internet]. Rio de Janeiro: IBGE; 2010 [citado 2016 junho 10]. Disponível em: http://www. ibge.gov.br/home/estatistica/populacao/censo2010/caracteristicas_religiao_ deficiencia/caracteristicas_religiao_deficiencia_tab_xls.shtm

18. Fundação Oswaldo Cruz. PROADESS: avaliação de desempenho do sistema de saúde brasileiro: indicadores para monitoramento. Rio de Janeiro: Laboratório de Informações em Saúde, Instituto de Comunicação e Informação em Ciência e Tecnologia, Fundação Oswaldo Cruz; 2011. Relatório final.

19. Botelho JF. Internações por condições sensíveis à atenção primária e fatores associados em Itaboraí, RJ [dissertação]. Rio de Janeiro: Escola Nacional de Saúde Pública Sérgio Arouca, Fundação Oswaldo Cruz; 2013 [citado 2016 junho 10]. Disponível em: http://www.proadess.icict.fiocruz.br/

20. Arruda JS, Costa JSD. Internações por condições sensíveis à atenção primária em Novo Hamburgo, Rio Grande do Sul. Rev Bras Med Fam Comunidade. 2017;12(39):1-11. http://dx.doi.org/10.5712/rbmfc12(39)1256.

21. Morimoto T, Costa JSD. Internações por condições sensíveis à atenção primária, gastos com saúde e Estratégia Saúde da Família: uma análise de tendência. Cien Saude Colet. 2017;22(3):891-900. http://dx.doi. org/10.1590/1413-81232017223.27652016. PMid:28300996.

22. Poli P No, Faoro NT, Prado JC Jr, Pisco LAC. Remuneração variável na Atenção Primária à Saúde: relato das experiências de Curitiba e Rio de Janeiro, no Brasil, e de Lisboa, em Portugal. Cien Saude Colet. 2016;21(5):1377-88. http://dx.doi.org/10.1590/1413-81232015215.02212016. PMid:27166888.

23. Souza DK, Peixoto SV, Souza DK, Peixoto SV. Estudo descritivo da evolução dos gastos com internações hospitalares por condições sensíveis à atenção primária no Brasil, 2000-2013. Epidemiol Serv Saude. 2017;26(2):285-94. http://dx.doi.org/10.5123/S1679-49742017000200006. PMid:28492770.

24. Brasil. Ministério da Saúde. Manual instrutivo do Programa Nacional de Melhoria do Acesso e da Qualidade da Atenção Básica [Internet]. Brasília; 2012. 62 p. (Série A. Normas e Manuais Técnicos) [citado 2016 junho 10]. Disponível em: http://189.28.128.100/dab/docs/publicacoes/geral/ manual_instrutivo_pmaq_site.pdf

25. Nóbrega-Therrien SM, Souza PMM, Pinheiro FMC, Castro VS. Formação para a Estratégia Saúde da Família na Graduação em Medicina. Rev Bras Educ Med. 2015;39(1):112-8. http://dx.doi.org/10.1590/1981$52712015 \mathrm{v} 39 \mathrm{n} 1 \mathrm{e} 02212012$.

26. Rehem TCMSB, Egry EY. Internações por Condições Sensíveis à Atenção Primária no Estado de São Paulo. Cien Saude Colet. 2011;16(12):4755-66. http://dx.doi.org/10.1590/S1413-81232011001300024. PMid:22124915.

27. Nedel FB. csapAIH: uma função para a classificação das condições sensíveis à atenção primária no programa estatístico R. Epidemiol Serv Saude. 2017;26(1):199-209. http://dx.doi.org/10.5123/S1679-49742017000100021.

Recebido em: Set. 06, 2017 Aprovado em: Maio 25, 2018 